See discussions, stats, and author profiles for this publication at: https://www.researchgate.net/publication/351730430

\title{
Robotics Laboratory within the Italian School-Work Transition Program in High Schools: A Case Study
}

Conference Paper · May 2021

CITATIONS

0

6 authors, including:

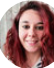

Gemma Carolina Bettelani

Università di Pisa

9 PUBLICATIONS 29 CITATIONS

SEE PROFILE

6

Riccardo Mengacci

Università di Pisa

9 PUBLICATIONS 17 CITATIONS

SEE PROFILE

Some of the authors of this publication are also working on these related projects:

TIGHT: Tactile InteGration for Humans and arTificial systems View project
READS

49

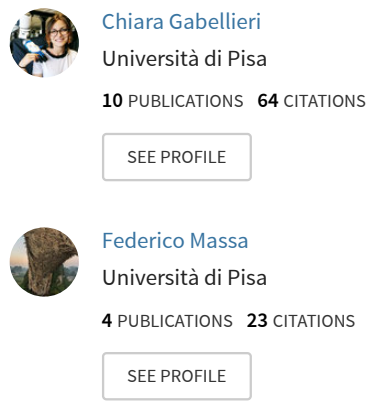




\title{
Robotics Laboratory within the Italian School-Work Transition Program in High Schools: A Case Study
}

\author{
Gemma C. Bettelani ${ }^{1}$, Chiara Gabellieri ${ }^{1}$ Riccardo Mengacci ${ }^{1}$, Federico Massa ${ }^{1}$, \\ Anna Mannucci ${ }^{2}$, and Lucia Pallottino ${ }^{1}$ \\ 1 Research Center E. Piaggio, Dept. Information Engineering of University of Pisa, Pisa, Italy \\ gemma.bettelanil@gmail.com \\ 2 Multi-Robot Planning and Control Lab, Örebro University, Sweden.
}

\begin{abstract}
This paper presents a robotics laboratory originated by the collaboration between the university and high school within the Italian school-work transition program. The educational objective of the proposed lab is twofold: 1) ease the transfer of robotic researchers' expertise into useful means for the students' learning; 2) teaching by practice the multidisciplinarity of robotics. We exploited the RoboCup Junior Race as a useful scenario to cover topics from 3D printing for fast prototyping to low-level and high-level controller design. An ad-hoc end-of-term student survey confirms the effectiveness of the approach. Finally, the paper includes some considerations on how general problems in the robotic and scientific community, such as gender issues and COVID-19 restrictions, can impact the educational robotics activities.
\end{abstract}

Keywords: Educational Robots, Lego Mindstorms NXT, High School, RoboCup Junior race, Italian work-school transition program

\section{Introduction}

The fast development of robotics is leading towards a greater and greater presence of robots in our society. One of the main sectors in which robots are showing their potential outside of industry and research is represented by education. As a matter of fact, educational robotics programs in schools are spreading worldwide [1].

The use of robots as a teaching method has been suggested to develop and improve not only students' academic skills (e.g., mathematics and geometry, coding, physics, etc.), but also to boost important soft skills [2], although further quantitative research might be needed to confirm this [3]. In particular, educational robotics is expected to help develop problem-solving, logic reasoning, creativity, linguistic skills, and social skills such as teamwork and interactions with peers $[4,5]$.

Robotics has been largely deployed in high-school curricula as well as in secondary and primary schools $[6,7]$. One of the most common tools that have been proven to be effective for introducing robotics to children and young students is LEGO Mindstorms ${ }^{3}$. Indeed, the pedagogical use of LEGO goes in the direction of learning through play, shown as a very efficient approach for students' learning [8].

\footnotetext{
${ }^{3}$ https://www.lego.com/it-it/themes/mindstorms
} 
As expected, while students are usually very enthusiastic about the use of robots at school, teachers may feel anxious about it and need adequate formation and, possibly, technical support during the lessons $[9,7,10]$.

Although many experiences have been conducted, robotic courses held by university robotic researchers are not common in most of the pre-university institutes. In this work, we describe a collaboration between the "Research Center E. Piaggio" of the University of Pisa and an Italian high school of the scientific and technological area ("ITI Galileo Galilei", Livorno, Italy). The collaboration was born in 2018 within the national program for school-work transition promoted by the Italian Ministry of Education (MIUR). The aim of the program is twofold: 1) putting into practice in realistic working environments the knowledge acquired at school; 2) helping the students in making a conscious decision on their future job or university career.

The objective of this paper is to share our experience that we think could be taken as a model by teachers in future robotics couses. Results of the students' end-of-term survey are reported and show positive feedback on the proposed approach. Finally, the paper also aims at discussing gender issues and COVID-19 restriction problems in the educational robotic field.

\section{Preliminary activities}

Before starting the robotics lessons in class, the students attended a general introduction about the activities of the "Research Center E. Piaggio" of the University of Pisa. In particular, a Professor of the University and the Ph.D. students that participated in the project (i.e., the authors) presented their research topics: humanoid robots, autonomous cars, drones, etc. The students had also the opportunity to visit the laboratories of the "Research Center E. Piaggio" and see real robots (e.g., KUKA arm [11], EGO robot [12]). During this visit, they could get an insight into real-world robotics applications. They also understood that robotics is not only limited to industrial robots but is a wide field that includes, for example, the realization of prosthetic robotic hands (e.g., the Pisa/IIT Softhand Pro [13]) and wearable haptics devices (e.g., the CUFF device [14] and the Skin-stretch device [15]), which are developed to restore the sense of touch in amputees.

These preliminary activities were of particular importance to introduce a large group of students to robotics and to give them a general overview of the multidisciplinarity of robotics, which includes mechanics, electronics, programming, automatic control and so on. Moreover, they could see how working in robotics can be an actual profession and had the chance to taste what working in the field of robotic research means (e.g., working in teams and finding people with different backgrounds such as bio-medical, mechanical, computer, automation, and even management engineers). Finally, the students who were most amazed by these activities decided to take part in the extracurricular robotics course being part of the work-school transition program. 


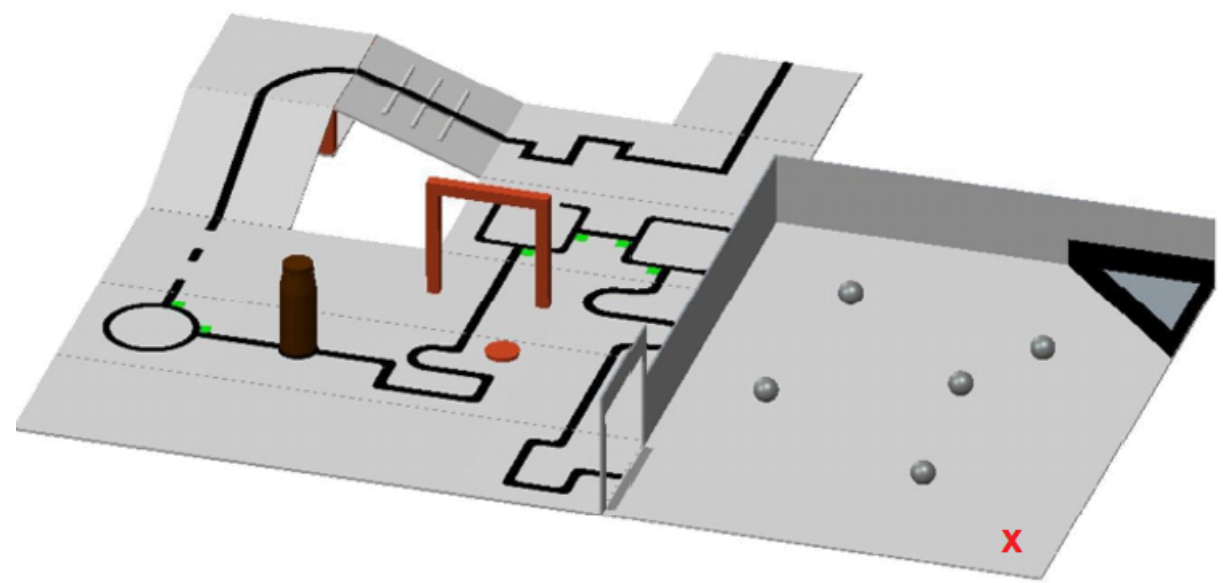

Fig. 1. Example of the race environment. On the left side: the black line that the robot had to follow (choosing the direction signaled by the green markers in the case of multiple options). On the right side: the rectangular "dangerous area" with the balls. The red cross indicates the other possible corner where the triangle could be placed

\section{The robotics classes}

The robotics laboratory within the Italian school-work transition program was scheduled into five lessons of four hours each, for a total of twenty hours. The aim of the lessons was to find strategies to finalize a "rescue mission" performed by an autonomous robot (Fig.1 shows an example of the race circuit). The "rescue mission" was part of the national program of the RoboCup Junior challenge ${ }^{4}$.

\subsection{The "rescue mission" in a nutshell}

The goal of the mission was to get as many points as possible before the time ran out. A mobile robot, positioned at the starting point, had to recognize and follow a twisty path, created with a black line, which was painted on a floor with many asperities (e.g., bumps, halls, obstacles). At the end of the black path, the robot found a silvery line that pointed out the entrance to a rectangular area: the "dangerous area" with the "victims", which were represented by balls. Particularly, the balls could be black ("dead victims") or silvery ("alive victims"). The robot had to pick up the balls and place them into a triangular area, which had a certain height with respect to the floor. The triangle could be positioned in one of the "dangerous area" corners that were in front of the robot when it entered the area. Particularly, the rescue of "alive victims" gave more points than the rescue of "dead victims". After rescuing all the "victims", the robot had to find a way to exit the "dangerous area".

\footnotetext{
${ }^{4}$ https://junior.robocup.org/
} 


\subsection{Lesson overview}

Eleven students attended the course and they were divided into groups of two or three students each. Each group had to realize and program its own robot, using the LEGO Mindstorms NXT kit. During the course, they had to find, by collaborating with the researchers and using the tools acquired during the lessons, strategies useful to accomplish the tasks of the race. Then, at the end of the course, the students were asked to compile a questionnaire related to the quality and the usefulness of the course.

In the following sections, we present the tools that the researchers gave to the students and some strategies that they found using them. The results of the questionnaire is discussed in Section 4.

\subsection{Proposed tools}

The students were already familiar with the ROBOTC programming language ${ }^{5}$ used to program their robots. Thus, at the beginning of the first lesson, they were able to give the robot's wheels the basic commands to move it backward or forward. For this reason, we decided to start the lessons with an overview of the sensors included in the LEGO Mindstorms kit that could be useful for the race. Particularly, we introduced the usage of the light sensor and the ultrasound sensor. During this lesson, we focused on the importance of sensor calibration. In the following lesson, we decided to introduce the students to the concept of closed-loop systems, comparing them to open-loop systems. Then, we explained a more advanced architecture: the PID controller. The choice was determined by the fact that some students were curious why sometimes their robots could not be able to reach exactly the desired position. After that, we decided to explain some basics of $3 D$ mechanical design, using the FreeCAD software ${ }^{6}$. This choice was motivated by the fact that it was allowed by the race rules to add additional 3D printed parts and by the fact that mechanical design is a very useful skill in robotics related to rapid prototyping. After the introduction to the aforementioned topics, we had a brainstorming session with each group to investigate possible strategies to accomplish the "rescue mission" tasks.

\subsection{Solutions and strategies}

In this section we report the most interesting strategies found by students' groups, brainstorming with the researchers.

- Black line following: to accomplish this task the students decided to use a light sensor positioned in the frontal part of the robot, between the wheels, and facing down. Thanks to the lesson related to sensor calibration, they were able to understand immediately that the values given by the sensor were strictly intertwined with the environment light condition. For this reason, they decided to change the threshold found for the black color every time the light conditions changed and they took note in order to do this during the race before the beginning of the "rescue mission".

\footnotetext{
5 https://www.robotc.net/

${ }^{6}$ https://www.freecadweb.org/
} 
- Identification of the triangle position: the students decided to use the ultrasound sensor (placed on the frontal side of the robot and facing ahead) to measure the robot distance from the side walls of the "dangerous area". In doing so, the robot was able to compute the center of the rectangular area in the transversal direction and to go there. After that, by measuring its distance from the frontal wall and from the corners of the room, the robot was able to understand in which corner of the area the triangle was. (see Fig. 2 for a clear explanation of all the steps)

- Pick up of the "victims": some students decided to design with the FreeCAD software a calliper (Fig. 3). They decided to control the calliper elevation by implementing the PID controller that the researchers explained during the related theoretical lessons.

- Coverage of the rectangular dangerous zone: The students decided to make the robot do a serpentine. If a light sensor, positioned at the base of the calliper, identified a ball, the robot moved forward until the wall, where the ball could be dragged in the calliper and then brought to the triangle. It is important to note that when the robot arrived at the triangle, the height of the calliper had to be controlled in order to lift the ball inside the triangle. For this task the students decided to not distinguish between "alive or dead victims" but only to understand if the robot had picked up a ball or not. (Fig. 4).

For the development of these strategies, the students have exploited multiple skills. Indeed, while the first and the third proposed strategies are related to the development of technical skills, the other two strategies are associated with problem-solving solutions.

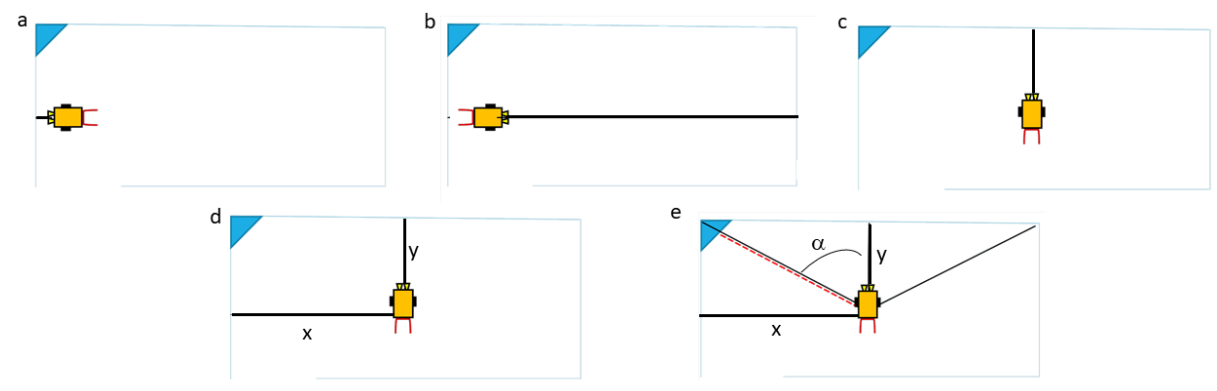

Fig. 2. Triangle position identification. The solutions proposed by the students articulated as follows: a) After the entrance of the robot in the rectangular area, the robot rotates counterclockwise and measures its distance from the wall on its left. b) The robot measures its distance from the wall on its right. $\mathbf{c}$ The robot can now compute the midpoint of the area width and when it places there, it computes its distance from the frontal wall. d-e) Known the distances computed in panel c, named $\mathbf{x}$ and $\mathbf{y}$, the robot can compute the angle $\alpha$. At this point, the robot turns counterclockwise and clockwise of $\alpha$. The triangle is placed in the corner where the measured distance robot-corner is smaller (dashed red line) 


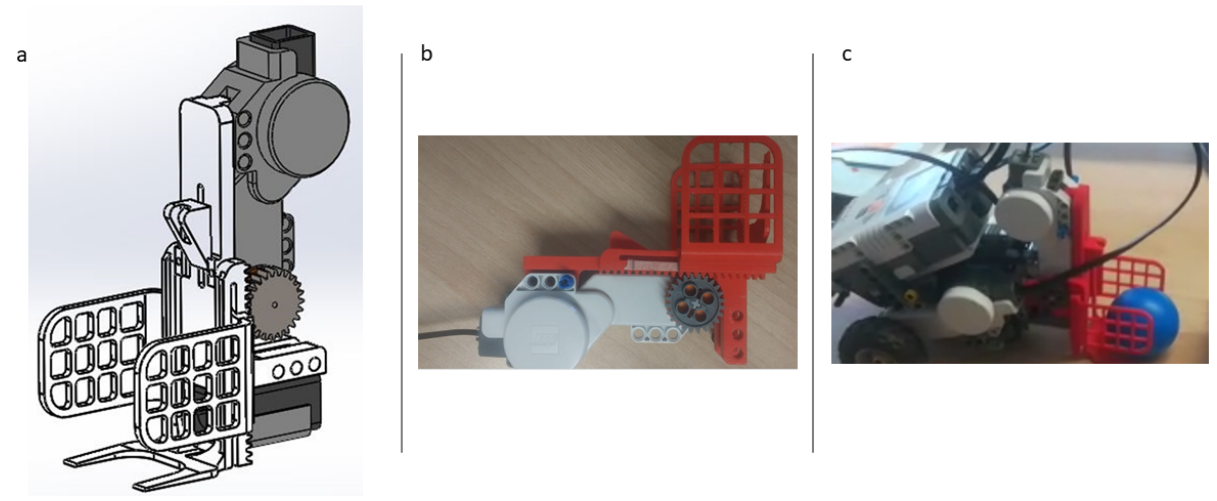

Fig. 3. Mechanical parts designed and realized by the students a) The calliper cad realized with the FreeCAD software. b) Lateral view of the calliper mounted on a LEGO motor. c) Calliper and motor mounted on the robot
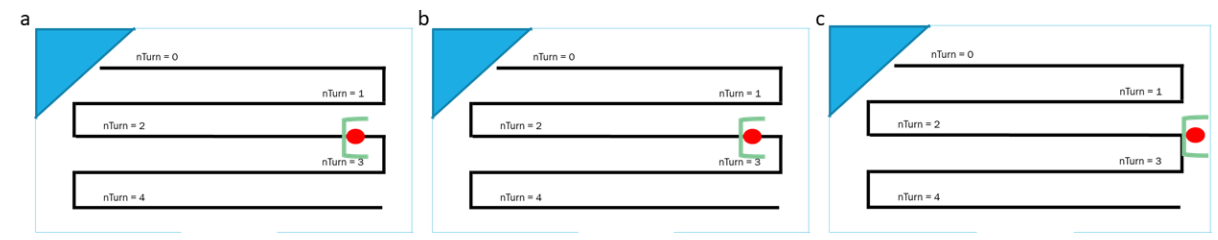

Fig. 4. Coverage strategy found by the students: when the robot identifies the position of the triangle, it memorizes that position and, in the meanwhile, it moves there. From the triangle position, the robot starts the serpentine and when it recognizes a ball, it drags it till the wall, so the ball can be wedged in the calliper and it can be brought to the triangle.

\section{Discussions}

The solutions proposed by the students were of course not the optimal solutions but the message that we tried to deliver was that often in robotics there is no unique solution. This is the reason why, in this field, it is important to work with others and share opinions and doubts. We also tried to show to the students that robotics is indeed multidisciplinary. For this reason, we introduced the 3D mechanical design and we invited Eng. Daniele Benedettelli, a freelance LEGO designer who presented to the students LEGONARO $^{7}$, a LEGO portrayer robot. During this lesson, the students had also the opportunity to be introduced to the concept of Artificial Intelligence that is becoming very intertwined with modern robotics.

Another important point that we would like to underline is that the students, during the implementation of the race solutions, were able to apply and understand the importance of the concepts explained during the lessons, such as the calibration of the sensors, the concept of closed-loop control and the 3D mechanical design. We think

\footnotetext{
${ }^{7}$ https://robotics.benedettelli.com/legonardo/
} 
that it was of particular relevance to have robotics researchers explaining these ideas. Indeed, robotics researchers naturally speak using the right contextual vocabulary and show great confidence towards topics they deal with regularly. Because of this, it comes pretty natural to us to teach these concepts, as we experience them daily. Teachers, on the other hand, usually lack deep robotics knowledge and practice, and can thus benefit from inputs coming from collaborations with universities. As mentioned before, this project was an experience of work-school transition, so it was essential that the students collaborated with experts in the robotic field. However, we would like to stress the importance that the teachers' knowledge of didactics and pedagogy would have in a curricular course. For this reason, in that case, the teachers should hold the course, but robotics professionals, such as robotics researchers, could provide substantial support to both students and teachers.

Moreover, we also think that this project was a great opportunity for both researchers and students. From one side, as said before, working on problem-solving with researchers was a good chance for the students to be introduced to the robotics world and more in general to the research world. On the other side, for us, this project was not only very satisfactory but it also gave us the opportunity to explain in a simple way concepts that we take for granted. Moreover, the students' course/project impressions are underlined by the results of the Likert scale questionnaire (Table 1) proposed at the end of the course. In particular, the students identified the project as useful (Q1), they considered the lessons well-explained (Q2) and they found that the lesson topics were sufficiently detailed (Q3). Then, the students considered it was useful to work in groups (Q4). They also considered that the course should have been longer (Q5). This last result is to be interpreted in light of the enthusiasm of the students. Indeed, some of them left a comment suggesting that they would have willingly done a longer stage.

Finally, we would like to discuss some important problems related to robotics. The first one is related to gender issues. The situation has improved considerably in recent years, with more women in the scientific fields. However, the work that needs to be done to highlight and resolve gender-related differences in STEM (Science, Technology, Engineering, and Mathematics) is still significant [16,17]. Indeed, it is necessary that females feel part of these fields as males do. Only male students participated in the laboratory in 2018. The laboratory was open to volunteers since it represented one possible choice among the opportunities offered within the school-work transition program. We want to stress the importance of the preliminary activities that we carried out (preliminary lessons and the visit to our laboratory) since they were aimed at a wider audience (a large number of students participated, females included, not only the ones involved in the laboratory activity). During the preliminary lessons and the visit to the "Research Center E. Piaggio", we explained that our course would be held by three female researchers and two male researchers, and all the students could directly see females at work in a robotics laboratory. Encouragingly, in 2019 one female participated in the course, and in 2020 two females were enrolled, even though the course was not held due to COVID-19 restrictions. It is important to underline that the high school "ITI Galileo Galilei" is a school mainly attended by males, so having two girls enrolled in the course can be considered a good result. We hope that we served as a positive 


\begin{tabular}{ll}
\hline Questions & mean \pm std \\
\hline Q1 It was useful having taken part in this project & $6.6 \pm 0.5$ \\
\hline Q2 The lessons were well explained & $6.7 \pm 0.5$ \\
\hline $\begin{array}{l}\text { Q3 The topics I was most interested in were suffi- } \\
\quad \text { ciently detailed }\end{array}$ \\
\hline \begin{tabular}{ll} 
Q4 It was useful working in groups & $6.2 \pm 0.7$ \\
\hline Q5 The entire course duration was appropriated & $4.4 \pm 0.7$
\end{tabular}
\end{tabular}

Table 1. Likert scale questions (from 1 to 7). 1 means that the students completely disagree with the question and 7 means that the students completely agree with the question.

role model to encourage also female students to approach robotics and that the greater female participation in consecutive years is a consequence.

The last point that is important to underline in this context is that educational scientific subjects have been really affected by COVID-19 restrictions. Indeed, organizing a laboratory tour and teaching a course like the one we held is difficult and not well manageable with the pandemic restrictions. However, the presentation of the laboratory activities could be held online. During pandemic, a virtual presentation of the laboratory activities of the Research Center E. Piaggio was tested for the Researchers' Night event and the experience was really immersive for the people that were at home (see the video of the event at this link https://www.youtube.com/watch?v=9QvTPpAReUg).

Then, to solve the problem of the robotics course, something could be achieved with funds to buy a laptop and a LEGO Mindstrorms kit for each student. The students could not collaborate during the lessons as they would have done in class, but they could at least try out their solutions on the robot. Moreover, it has also been suggested [18] that simulators represent the most suitable solution to easily carry on robotics classes within the restrictions imposed by the recent social distancing. Indeed, if the school has not enough funds to buy a huge number of robots, each student could use the simulator to test his/her strategies and then the teacher could try the solutions on the real robot during a video lesson. Furthermore, the target simulator should be easy-to-use, accurate enough for the students to test their solutions reliably, and moderately priced. Finally, to solve the problem related to the printing of the 3D mechanical parts, the school could rely on online platforms that offer $3 \mathrm{D}$ printing and shipping services (e.g., $3 \mathrm{Dhubs}{ }^{8}$ or Fab Labs of the city)

\section{Acknowledgments}

We would like to thank Professor Cristina Tani and Professor Giorgio Meini of the high school "ITI Galileo Galilei", Livorno, Italy, who gave us the possibility to undertake this great experience. We also thank Eng. Daniele Benedettelli, who took part in this course with his great experience with LEGO usage and his enthusiasm.

\footnotetext{
${ }^{8}$ https://www.3dhubs.com/
} 


\section{References}

1. David P Miller and Illah Nourbakhsh. Robotics for education. In Springer handbook of robotics, pages 2115-2134. Springer, 2016.

2. Lai Poh Emily Toh, Albert Causo, Pei-Wen Tzuo, I-Ming Chen, and Song Huat Yeo. A review on the use of robots in education and young children. Journal of Educational Technology \& Society, 19(2):148-163, 2016.

3. Fabiane Barreto Vavassori Benitti. Exploring the educational potential of robotics in schools: A systematic review. Computers \& Education, 58(3):978-988, 2012.

4. Samuel Blanchard, Viktor Freiman, and Nicole Lirrete-Pitre. Strategies used by elementary schoolchildren solving robotics-based complex tasks: Innovative potential of technology. Procedia-Social and Behavioral Sciences, 2(2):2851-2857, 2010.

5. Isabelle ML Souza, Wilkerson L Andrade, Lívia MR Sampaio, and Ana Liz Souto O Araujo. A systematic review on the use of lego ${ }^{\circledR}$ robotics in education. In 2018 IEEE Frontiers in Education Conference (FIE), pages 1-9. IEEE, 2018.

6. David Scaradozzi, Laura Sorbi, Anna Pedale, Mariantonietta Valzano, and Cinzia Vergine. Teaching robotics at the primary school: An innovative approach. Procedia - Social and Behavioral Sciences, 174:3838 - 3846, 2015. International Conference on New Horizons in Education, INTE 2014, 25-27 June 2014, Paris, France.

7. Ahmad Khanlari and Fatemeh Mansourkiaie. Using robotics for stem education in primary/elementary schools: Teachers' perceptions. In 2015 10th International Conference on Computer Science \& Education (ICCSE), pages 3-7. IEEE, 2015.

8. Soumela Atmatzidou, Iraklis Markelis, and Stavros Demetriadis. The use of lego mindstorms in elementary and secondary education: game as a way of triggering learning. In International Conference of Simulation, Modeling and Programming for Autonomous Robots (SIMPAR), Venice, Italy, pages 22-30. Citeseer, 2008.

9. Christina Chalmers. Robotics and computational thinking in primary school. International Journal of Child-Computer Interaction, 17:93-100, 2018.

10. Dimitris Alimisis. Robotics in education \& education in robotics: Shifting focus from technology to pedagogy. In Proceedings of the 3rd International Conference on Robotics in Education, pages 7-14, 2012.

11. Rainer Bischoff, Johannes Kurth, Günter Schreiber, Ralf Koeppe, Alin Albu-Schäffer, Alexander Beyer, Oliver Eiberger, Sami Haddadin, Andreas Stemmer, Gerhard Grunwald, et al. The kuka-dlr lightweight robot arm-a new reference platform for robotics research and manufacturing. In ISR 2010 (41st international symposium on robotics) and ROBOTIK 2010 (6th German conference on robotics), pages 1-8. VDE, 2010.

12. Gianluca Lentini, Alessandro Settimi, Danilo Caporale, Manolo Garabini, Giorgio Grioli, Lucia Pallottino, Manuel G Catalano, and Antonio Bicchi. Alter-ego: a mobile robot with a functionally anthropomorphic upper body designed for physical interaction. IEEE Robotics \& Automation Magazine, 26(4):94-107, 2019.

13. Sasha B Godfrey, Matteo Bianchi, Kristin Zhao, Manuel Catalano, Ryan Breighner, Amanda Theuer, Karen Andrews, Giorgio Grioli, Marco Santello, and Antonio Bicchi. The softhand pro: Translation from robotic hand to prosthetic prototype. In Converging Clinical and Engineering Research on Neurorehabilitation II, pages 469-473. Springer, 2017.

14. Simona Casini, Matteo Morvidoni, Matteo Bianchi, Manuel Catalano, Giorgio Grioli, and Antonio Bicchi. Design and realization of the cuff-clenching upper-limb force feedback wearable device for distributed mechano-tactile stimulation of normal and tangential skin forces. In 2015 IEEE/RSJ International Conference on Intelligent Robots and Systems (IROS), pages 1186-1193. IEEE, 2015. 
15. Nicoletta Colella, Matteo Bianchi, Giorgio Grioli, Antonio Bicchi, and Manuel G Catalano. A novel skin-stretch haptic device for intuitive control of robotic prostheses and avatars. IEEE Robotics and Automation Letters, 4(2):1572-1579, 2019.

16. Katherina Kuschel, Kerstin Ettl, Cristina Díaz-García, and Gry Agnete Alsos. Stemming the gender gap in stem entrepreneurship-insights into women's entrepreneurship in science, technology, engineering and mathematics. International Entrepreneurship and Management Journal, 16(1):1-15, 2020.

17. Bettina J Casad, Jillian E Franks, Christina E Garasky, Melinda M Kittleman, Alanna C Roesler, Deidre Y Hall, and Zachary W Petzel. Gender inequality in academia: Problems and solutions for women faculty in stem. Journal of neuroscience research, 99(1):13-23, 2021.

18. Bryan Bergeron. Online stem education a potential boon for affordable at-home simulators. SERVO Magazine, 2, 2020. 